\title{
Eta Carinae and the Luminous Blue Variables
}

\author{
Jorick S. Vink
}

\begin{abstract}
We evaluate the place of Eta Carinae ( $\eta$ Car) among the class of luminous blue variables (LBVs) and show that the LBV phenomenon is not restricted to extremely luminous objects like $\eta$ Car, but extends luminosities as low as $\log \left(L / L_{\odot}\right)$ $\sim 5.4$ - corresponding to initial masses $\sim 25 M_{\odot}$, and final masses as low as $\sim 10$ $15 M_{\odot}$. We present a census of S Doradus variability, and discuss basic LBV properties, their mass-loss behavior, and whether at maximum light they form pseudophotospheres. We suggest that those objects that exhibit giant $\eta$ Car-type eruptions are most likely related to the more common type of S Doradus variability. Alternative atmospheric models as well as sub-photospheric models for the instability are presented, but the true nature of the LBV phenomenon remains as yet elusive. We end with a discussion of the evolutionary status of LBVs - highlighting recent indications that some LBVs may be in a direct pre-supernova state, in contradiction to the standard paradigm for massive star evolution.
\end{abstract}

\section{Introduction}

Luminous Blue Variables (LBVs) are evolved, luminous hot stars that experience eruptions or outbursts and episodes of enhanced mass loss. During outburst they appear to make transits in the HR Diagram (HRD) from their normal hot quiescent state to lower temperatures. The LBVs include a number of famous stars such as $\mathrm{P}$ Cyg, S Dor, R127 and AG Car. Eta Car is often described as an LBV, although it is a more extreme example owing to its giant eruption.

During the late 1970's, it was recognized that the distribution of the most luminous hot stars on the HRD defines a locus of declining luminosity with decreasing temperature [48, 54, 114]. Together with the fairly tight upper luminosity limit of

Jorick S. Vink

Armagh Observatory, College Hill, Armagh BT61 9DG, Northern Ireland, e-mail: jsvearm.ac.uk 
the yellow and red supergiants at $\log \left(L / L_{\odot}\right) \approx 5.8$ [48], this indicated that the most massive stars $\left(M>60 M_{\odot}\right)$ do not evolve to cooler temperatures: the HumphreysDavidson (HD) limit [48]. Humphreys and Davidson [48, 49] suggested that high mass-loss episodes, represented by stars like $\eta$ Car, P Cyg, S Dor and the HubbleSandage variables in M31 and M33 [47], prevented the evolution of the most massive stars to cooler temperatures. With this addition of a post-main sequence period of high mass loss $\left(10^{-5}-10^{-3} M_{\odot} \mathrm{yr}^{-1}\right)$ the evolutionary tracks of the most massive stars were shown to turn bluewards, towards the core He-burning Wolf-Rayet (WR) phase. During the WR phase, stars are anticipated to explode as supernovae ( $\mathrm{SNe}$ ) type Ib/c. However, at the end of this chapter, we challenge the canonical view that LBVs are always transitional between the $\mathrm{O}$ and WR stars, and suggest that some massive stars may already suffer their final explosion during or at the end of the LBV phase. One of the most relevant questions is therefore do LBVs explode?

The term LBV was introduced to describe the diverse group of unstable evolved hot stars in the upper HRD. Today we distinguish between more than one type of LBV [50]: (i) the normal LBV variability cycles with visual magnitudes changes of 1-2 magnitudes at essentially constant luminosity - on timescales of years to decades - represented by the prototype of the class, S Dor, in the Large Magellanic Cloud, and (ii) the giant eruption LBVs represented by $\eta$ Car and P Cyg with visual magnitudes changes of 3 magnitudes or more, during which the total bolometric luminosity increases [50, 52].

In this chapter, we focus on the $S$ Dor-type variables and their transits in the HRD, the prime characteristics of the LBV class. Roughly 30 massive stars in the Galaxy $(\simeq 10)$ and Local Group $(\simeq 20)$ are known to be S Dor variables. By contrast, only two Galactic objects have been discovered to exhibit giant $\eta$ Car-like eruptions: $\eta$ Car itself and P Cygni, which suddenly appeared at naked-eye visibility in 1600 . Due to their high luminosities at maximum, a significant number of LBV-like non-terminal eruptions have been discovered in external galaxies. Most are typical of giant eruptions whilst some appear to be more similar to S Dor type variables. These extragalactic LBVs are sometimes referred to as "SN imposters" (see the contribution by Van Dyk and Matheson, this volume).

The circumstellar nebulae seen around many Galactic and MC LBVs [85, 132] may also have resulted from giant $\eta$ Car eruptions, although stationary winds from prior evolutionary phases may constitute an alternative scenario for their creation [32]. Given the association of many LBVs with nebulae and ejecta and the close proximity of both the $\mathrm{S}$ Dor variables and the $\eta$ Car-like variables to the Eddington limit, it is often suggested (though not proven) that their instabilities represent different manifestations of the same underlying evolutionary state. The fact that the giant eruptor P Cyg is also subject to small amplitude S Dor variability [24, 77] and that $\eta$ Car's second outburst (during 1888-1895) was like that of a normal S Dor variable [52] lends support to this possibility.

We emphasize, however, that neither the $\eta$ Car-type eruptions, nor the S Dor variations are understood. Worse still, we do not know whether all LBVs are subject to both types of variability, or in which order they may occur. Two pertinent questions 
are thus whether " $\eta$ Car is unique among the LBVs?" and "what is the root cause of the S Doradus variations?"

\section{Basic properties of LBVs}

Variability LBVs show significant spectroscopic and photometric variability on timescales of years (short S Dor phases) to decades (long S Dor phases, cf. van Genderen [123]). For completeness, we note that LBVs also show smaller amplitude "micro" variability, on shorter timescales (weeks, months), but this aspect of their variability is also a common feature of supergiants in general (see [72, 68] and references therein).

Luminosities The classical LBVs have $\log \left(L / L_{\odot}\right)$ larger than 5.8 with bolometric magnitudes in the range $M_{\text {bol }}-9$ to -11 . There is an apparent "gap" in their luminosities just below $\log \left(L / L_{\odot}\right)=5.8$, and a separate group of less luminous LBVs with $\log \left(L / L_{\odot}\right)=5.4-5.6$, corresponding to bolometric magnitudes in the range $M_{\text {bol }} \simeq-8$ to $\simeq-9$ [50]. We note that this separation in luminosity (see Fig 11) may not be real, but due to small number statistics. As the less luminous LBVs are below the HD limit, they have presumably been red supergiants where they may already have shed a lot of mass. As a result, they may be equally close to their Eddington limit as the classical LBVs.

One of the most important properties of LBVs is that they appear to be close to the Eddington limit for stability against radiation pressure for their luminosities and current masses (see the subsection on stellar masses). The Eddington luminosity $L_{\mathrm{E} d d}$ is defined:

$$
L_{\mathrm{Edd}}=\frac{4 \pi c G M}{\kappa_{\mathrm{F}}}
$$

where $\kappa_{\mathrm{F}}$ is the flux-mean opacity and $M$ is the total mass of the star.

The dimensionless Eddington parameter $\Gamma$ is defined as the ratio of radiative acceleration to gravitational acceleration (disregarding sign):

$$
\Gamma=\frac{\kappa_{\mathrm{F}} L}{4 \pi c G M}=\left(7.66 \times 10^{-5} \mathrm{~g} \mathrm{~cm}^{-2}\right) \kappa_{\mathrm{F}}\left(\frac{L}{L_{\odot}}\right)\left(\frac{M}{M_{\odot}}\right)^{-1} .
$$

If opacity is entirely due to Thomson scattering by free electrons, as often assumed in this connection, then $\kappa_{\mathrm{F}} \approx 0.3 \mathrm{~cm}^{2} \mathrm{~g}^{-1}$; but this depends somewhat on chemical composition and ionization state.

Temperatures LBV temperatures are not only time-variable, but accurate $T_{\text {eff }}$ determination requires sophisticated non-LTE spectral analyses for hot stars with extended envelopes and winds. Furthermore, it should be noted that for extreme objects like $\eta$ Car we cannot even estimate the radius based on $T_{\text {eff }}$ as the wind is 


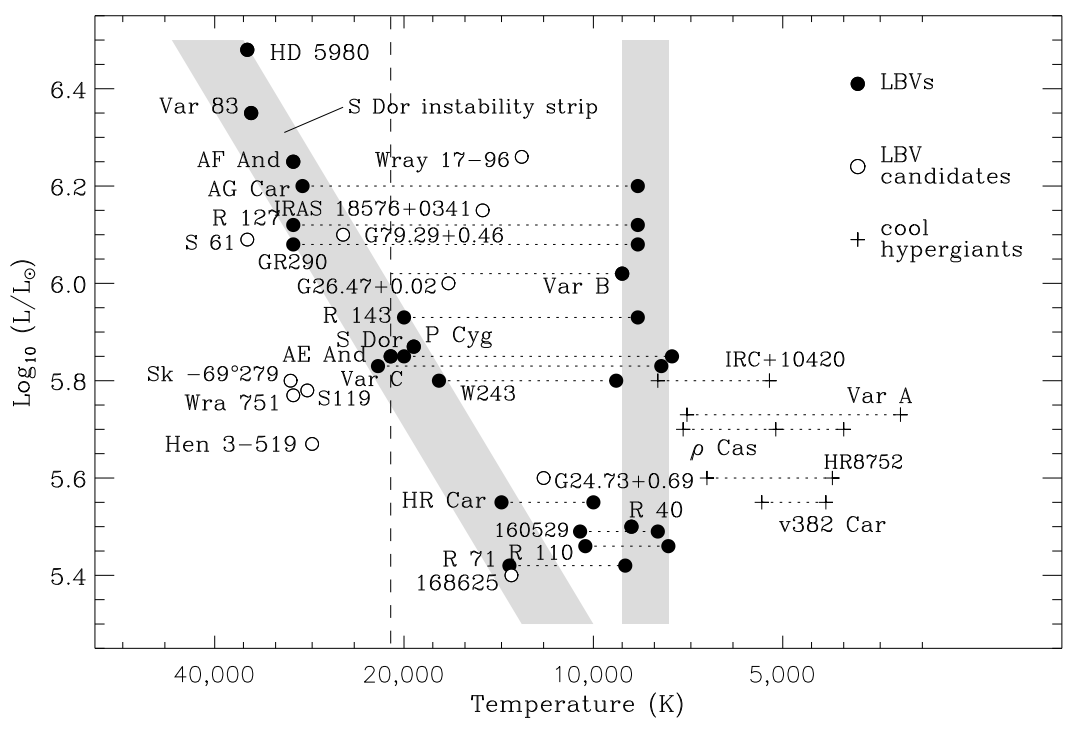

Fig. 1 The location of the LBVs (black circles) and candidates (open circles) in the HertzsprungRussell Diagram. The cool yellow hypergiants are indicated with pluses. The slanted and vertical grey bands represent visual minimum and maximum respectively. The dashed vertical line at $21000 \mathrm{~K}$ indicates the location of the bi-stability jump (see Sect. 3. The figure has been adapted from Smith et al. [106] and is similar to e.g. Fig. 9 in [50].

optically thick. In such a case, $T_{\text {eff }}$ refers to the position in the wind where $R_{\mathrm{ph}}>$ $R_{\text {sonic }}$ (see below for a more extended discussion).

Fig. 11 shows the confirmed and candidate LBVs on a schematic HRD with their transits (the dotted horizontal lines) between quiescence and their outburst state or visual maximum. At quiescence, or visual minimum, they appear to lie along a fairly narrow slanted band: the "S Dor instability strip" [135]. Their effective temperatures vary from over $30000 \mathrm{~K}$, corresponding to spectral type late O/early $\mathrm{B}$, for the luminous classical LBVs to only $\simeq 15000 \mathrm{~K}$, corresponding to late B spectral types, for the less luminous LBVs. Objects from both subgroups transit redwards to $T_{\text {eff }}$ not higher than $\simeq 8000 \mathrm{~K}$, corresponding to spectral types A to F, when they are "in outburst" or visual maximum. Wolf [135] noted that the amplitudes of the S Dor excursions become larger with increasing luminosity, and suggested that the most luminous LBVs could be cosmological distance indicators. Normal blue supergiants are also observed in and to the right of the $\mathrm{S}$ Dor instability strip, but they may not be close to their Eddington limit and may therefore not be subjected to the instability that characterizes LBVs. The cool or yellow hypergiants (YHGs) just below the HD limit [48, 50] are included as these might somehow represent the "missing" LBVs [106] - a possibility which will be discussed further in Sect. 3. 
Membership Whether a particular blue hypergiant is a member of the select group of LBVs (see Table 11) is often a matter of debate (see [50, 12, 79]). In general, spectral resemblance to the P Cygni type spectrum of a known LBV is not a sufficient criteria for a star to be called an LBV. For this reason we include a group of LBV candidates (LBVc) listed in Table 2 To officially qualify as an LBV, an object should at least have shown the combination of spectral-type/photometric variations characteristic of S Dor-type variability due to changes in the star's apparent temperature. Spectral variability, e.g. due to mass-loss changes, on its own is not a criterion, as almost all massive stars would qualify. Changes in photometric color could be the result of $T_{\text {eff }}$ changes, however obscuration by circumstellar dust may also give rise to color changes. Finally, the association of a supergiant with a circumstellar nebula is also not a criterion for an object to be added to the list of LBVs. The famous pistol star and LBV 1806-20 are thus strictly not LBVs. These objects remain candidates until they have shown the characteristic spectral-type variations.

Table 1 The confirmed LBVs. See [121, 80, 75] for the latest additions.

\begin{tabular}{|c|c|c|c|c|}
\hline \multicolumn{4}{|l|}{ Galaxy: } & HD 160529 \\
\hline HD 168607 & FMM 362 & AFG1 2298 & $\mathrm{G} 24.73+0.69$ & W1-243 \\
\hline $\begin{array}{l}\text { GCIRS } 34 \mathrm{~W} \\
L M C \text { : }\end{array}$ & Wra 751 & G0.120 - 0.048 & & \\
\hline S Dor & R71 & R 110 & R 116 & R127 \\
\hline $\begin{array}{l}\mathrm{R} 143 \\
\text { SMC: }\end{array}$ & HD 269582 & HD 269929 & & \\
\hline $\begin{array}{l}\mathrm{R} 40 \\
\text { M31: }\end{array}$ & HD 5980 & & & \\
\hline $\begin{array}{l}\text { AE And } \\
\text { M33: }\end{array}$ & AF And & Var A-1 & Var 15 & \\
\hline $\begin{array}{l}\text { Var B } \\
\text { N93351 } \\
\text { M81: }\end{array}$ & Var C & Var 2 & Var 83 & GR 290 \\
\hline $\begin{array}{l}\text { I } 1 \\
\text { M101: }\end{array}$ & I 2 & I 3 & & \\
\hline $\begin{array}{l}\mathrm{V} 1 \\
N G C 2403:\end{array}$ & V 2 & V 10 & & \\
\hline $\begin{array}{l}\text { V } 12 \\
N G C \text { 1058: } \\
\text { SN } 1961 \mathrm{~V}\end{array}$ & V 22 & V 35 & V 37 & V38 \\
\hline
\end{tabular}

When LBVs are discussed in an evolutionary context, we should be aware that the LBV phenomenon is likely to be intermittent and that part of the population might be dormant. Disregarding this could lead to incorrect interpretations with respect to their relative numbers and their evolutionary state. When we discuss the LBV phenomenon, however, we should only include the confirmed LBVs.

Abundances As massive stars evolve on the main sequence their atmospheric abundances are expected to undergo a transition in chemical abundances from solar $\mathrm{He}$ 
Table 2 The LBV candidates. See [116 40] 9] for the latest additions

\begin{tabular}{lllll}
\hline Galaxy: & & & & \\
Cyg OB2\#12 & Pistol star & HD 168625 & HD 326823 & HD 316285 \\
He3-519 & HD 80077 & $\zeta^{1}$ Sco & MWC 314 & MWC 930 \\
AS 314 & G25.5+0.2 & G79.29+0.46 & G26.47+0.02 & Wra17-96 \\
WR102ka & LBV1806-20 & Sher 25 & W51 LS1 & GCIRS 16NE \\
GCIRS 16C & GCIRS 16SW & GCIRS 16NW & GCIRS 33SE & \\
MN112 & & & & \\
LMC: & & & & \\
R 4 & R 66 & R 74 & R 78 & R 81 \\
R 84 & R 85 & R 99 & R 123 & R 128 \\
R 149 & S 18 & S 22 & S 61 & S 119 \\
S 134 & & & & \\
\hline
\end{tabular}

and $\mathrm{CNO}$ abundances to He-enriched and nuclear-equilibrium $\mathrm{CNO}$ abundances (with $\mathrm{N}$ enhanced, $\mathrm{C} / \mathrm{O}$ depleted) [74]. For massive stars with $\simeq 60 M_{\odot}$, this transition occurs rather rapidly after about 2 Myr of evolution [137]. LBVs show a wide span of CNO ratios. Davidson et al. [19] showed that the ejecta around $\eta$ Car are enhanced in both nitrogen and helium, and Pasquali et al. [90] found that the shell ejected by the LBVc HD 168625 is also N-enriched compared to the interstellar medium by a factor of several. Smith et al. [102] studied a sample of LBV nebulae and found the ejecta to be generally N-enhanced, although most nebulae have not reached abundances characteristic of $\mathrm{CNO}$ equilibrium.

The origin of the circumstellar nebulae associated with some LBVs however is uncertain. Photospheric abundance measurements might therefore provide a more direct means to constrain the LBV evolutionary state. These abundances however depend not only on the details of the atomic physics but also on the complexities of non-LTE spectral analyses. For example, Hillier et al. [46] showed that the H/He ratio in the wind of $\eta$ Car is ambiguous due to a strong coupling with the massloss rate. Eta Car however, could be an exception with its huge luminosity and high mass-loss rate. Najarro et al. [83] studied the atmospheric He abundance of P Cygni and quoted a best value of $\mathrm{n}(\mathrm{He}) / \mathrm{n}(\mathrm{H})$ of 0.3 , corresponding to a mass fraction $\mathrm{Y}$ $=0.63$. However, they noted a huge uncertainty due to the trade-off in ionization and $\mathrm{He}$ abundance and provided a $\mathrm{He}$ abundance range of $\mathrm{n}(\mathrm{He}) / \mathrm{n}(\mathrm{H})=0.25-0.55$. Hillier et al. [45] also ruled out a solar He abundance for the extreme P Cygni star HD 361285, but admitted that the uncertainty in $n(\mathrm{He}) / \mathrm{n}(\mathrm{H})$ could be as large as a factor 20! Evidence for advanced CNO processing was found by Lennon et al. [71] for the LBV R71, but this is not a well-established result for LBVs in general. Although most LBVs have He and $\mathrm{N}$ enhanced atmospheres, it seems unlikely that all of them have reached equilibrium $\mathrm{CNO}$ values in their outer atmospheres.

Stellar masses Masses are most accurately determined using detached binary systems. However, almost all LBVs are single. Eta Car is the best-known exception to this rule, with its 5.5y periodicity [134, 16] attributed to a companion. The current 
mass of $\eta$ Car is thought to be at least $90 M_{\odot}$, to avoid violating the Eddington Limit at its high luminosity (see Davidson this volume, Owocki and Shaviv this volume).

LBVs with $\log \left(L / L_{\odot}\right)>5.8$, are usually assumed to be evolved from the most massive stars with $M>50 M_{\odot}$ (e.g. [100]). Mass measurements for these highluminosity objects are scarce and uncertain. Pauldrach \& Puls [93] quote a mass of $23 M_{\odot}$ for P Cyg. Vink \& de Koter [127] estimated LBV masses from time-variable mass-loss rates and found a mass of $35 M_{\odot}$ for AG Car. These results suggest that classical LBVs have already lost a significant fraction of their initial mass probably through the combined effects of line-driven mass loss during the OB and LBV phase, and/or through prior major eruptions. The quoted mass estimates are highly model dependent. For instance, wind clumping was not considered for the mass estimate of AG Car. However, if these objects have lost half their initial mass, their $L / M$ ratio is quite large, with $\Gamma_{e} \simeq 0.5$, and they are close to the Eddington Limit for their luminosities.

The initial masses of the less luminous LBVs, $\log \left(L / L_{\odot}\right) \simeq 5.4$, is of the order $M \sim 25 M_{\odot}$; e.g. [100]). Comparing this mass with current-day mass estimates of $M$ $\sim 12 M_{\odot}$ for R71 [70], $M \sim 10 M_{\odot}$ for R110 [112], and $M \sim 13 M_{\odot}$ for HD 160529 [115], suggests that these low-luminosity LBVs may already have lost more that half of their initial mass (e.g. during a prior RSG phase). The low-luminosity LBVs are equally close to their Eddington limit with $\Gamma_{e} \simeq 0.6$. All of these empirical mass estimates are uncertain by at least a factor two.

Interestingly, Martins et al. [78] recently reported that the LBVc GCIRS 16SW may be an eclipsing binary, with both components weighing $\simeq 50 M_{\odot}$. If it is a genuine LBV, it may be a key object for constraining massive star evolution models, because current models with rotation [81] suggest such massive objects will not pass through the LBV phase at all during their evolution.

\section{Mass-loss properties - do LBVs form pseudo-photospheres?}

With its current mass-loss rate of order $10^{-3} M_{\odot} \mathrm{yr}^{-1}$ it is clear that $\eta$ Car has formed an optically-thick wind and a pseudo-photosphere, but whether the S Dortype variables have optically thick winds is less well established. It has been suggested that the temperature changes during $\mathrm{S}$ Dor cycles do not represent true stellar temperature changes, but are due to the formation of the dense, optically-thick wind. As the mass-loss rate increases, the effective photosphere of the moves out into the wind, and the apparent effective temperature of the star drops, whilst the apparent stellar radius increases - without an actual expansion of the star [3, 59, 18]. Davidson [18] also showed that the minimum temperature the wind can achieve as the mass loss increases is $\sim 7000 \mathrm{~K}$, in rough agreement with the apparent temperatures of the LBVs at visual maximum.

Normal OB stars have winds that are optically thin in the continuum and we see through the entire wind - down to the photosphere. In other words, the wind is formed outside the photosphere. However, when the mass-loss rates increases, 
the wind becomes less transparent, and the photosphere from which the optical light originates is now at larger radii. When the wind has become optically thick, the photosphere is formed above the sonic velocity. In other words, the wind is accelerated "inside" the photosphere - forming an opaque wind with $R_{\mathrm{ph}}>R_{\text {sonic }}$.

Leitherer et al. [70] and de Koter et al. [27] performed detailed NLTE modelling of this process, showing that the extent of a pseudo-photosphere that results from increased mass loss is relatively modest in comparison to that of a WR star. The conclusion was that the underlying LBV radius itself must become larger due to an as yet unidentified sub-photospheric mechanism. Furthermore, it became clear that empirical mass-loss rate during S Dor redward excursion do not always increase during outburst [69, 127]. More recently, Smith et al. [106] showed that pseudophotospheres might be feasible under certain special circumstances discussed later in Sect 3.3 Before we provide a more detailed account of the possibility of pseudophotosphere formation, we give an overview of our current knowledge of stationary LBV mass loss. The radiative forces that may be responsible for giant eruptions are discussed elsewhere (Owocki and Shaviv, this volume).

\subsection{Observed mass-loss rates}

Analysis of the blue-shifted absorption components in the P-Cygni profiles of strong emission lines such as $\mathrm{H} \alpha$ is commonly used to determine mass-loss rates from massive stars.

Terminal velocities The terminal velocities of $L B V$ winds measured from the blue edge of the P Cygni absorption component are in the range $100-250 \mathrm{kms}^{-1}$, with $\eta$ Car having a $v_{\infty}$ of $\sim 500 \mathrm{kms}^{-1}$ (cf. [69]). These wind velocities are significantly lower than those of normal OB supergiants, which have $v_{\infty} \sim 1000-3000 \mathrm{~km} \mathrm{~s}^{-1}$. The mass-loss rates of LBVs are also a factor of 10-100 larger than those of normal supergiants, so their wind densities, $\rho(r)=\dot{M} / 4 \pi r^{2} v(r)$, are much higher, giving the line profiles their characteristic P Cygni shapes. As the LBV mass-loss rate is variable, some LBVs exhibit profile shape changes and variability in the absorption profile, such as the split blue-shifted absorption components seen in the $\mathrm{H} \alpha$ line of AG Car (see Fig. 2) and other LBVs such as R 127 [111, 110]. P Cyg, R 66, and HD 160529 also exhibit shell components in their Fe II lines (cf. [63] for P Cyg). The split $\mathrm{H} \alpha$ absorption components in LBVs have recently been proposed to be the result of an abrupt bi-stability jump [34].

Mass-loss rates Mass-loss rates of most LBVs have been determined using nonLTE models such as CMFGEN [44]. The very high mass-loss rate for $\eta$ Car $\left(3 \times 10^{-3}\right.$ $M_{\odot} \mathrm{yr}^{-1}$ ) has been determined by several methods using radio, mm-wavelength, and Hubble Space Telescope data [133, 14, 20], and the non-LTE model results for $\eta$ Car [46] yield a similar answer. Sophisticated NLTE mass-loss determinations for other LBVs include a value of $3 \times 10^{-5} M_{\odot} \mathrm{yr}^{-1}$ for the extreme twin of P Cyg: HD 316285 


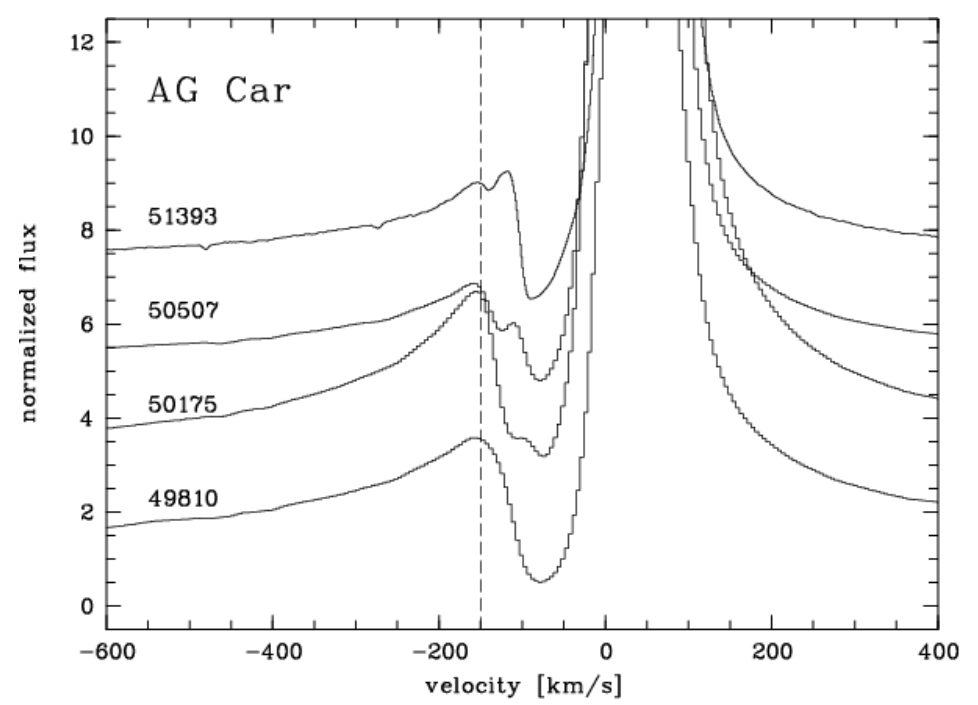

Fig. 2 The appearance of double-split absorption components in $\mathrm{H} \alpha$ in the spectrum of AG Car during the years 1996 to 1999. The spectra are marked in Julian days. The figure has been taken from Stahl et al. 113.

[45], while Najarro et al. [83] derived $3 \times 10^{-5} M_{\odot} \mathrm{yr}^{-1}$ for P Cyg itself, using similar analysis tools. These results are all much higher than for normal OB supergiants of comparable temperatures.

The non-LTE model based mass-loss determinations however are based on assumed spherical winds, but the wind of $\eta$ Car, is bipolar [105, 122]. Whether nonspherical winds have a large effect on the mass-loss determination, remains to be shown. A more serious assumption may be homogeneity. Davies et al. [21] performed a linear spectropolarimetry survey of Galactic and MC LBVs and found large line polarizations in over half of their survey targets. This is a higher incidence of polarization line effects than in $\mathrm{O}$ and WR stars where asphericities of resp. $\sim 25 \%$ [42, 130] and $\sim 15 \%$ [41, 126] have been reported. Rather than attributing the polarization to large-scale axi-symmetry (e.g. [101]), Davies et al. [21] attribute the linear polarization of LBVs like AG Car to wind clumping, because the position angle in the polarization was shown to vary significantly, as was the case for PCyg [84]. A similar conclusion regarding overall sphericity of LBV winds was drawn by Guo \& $\mathrm{Li}[38]$ on the basis of modelling LBV continuum energy distributions.

The LBV polarization variability implies that the clumps must arise close to the photosphere, and many small clumps predominate over a few larger ones [22]. The quantitative implications of wind clumping for the absolute mass-loss rate of LBVs and in early-type stars, in general, have yet to be established [39].

Although most LBVs have been monitored photometrically, only a handful have been subject to quantitative spectroscopic analysis at various epochs, and mass-loss rates have rarely been reported for different S Dor phases. In this respect, AGCar 


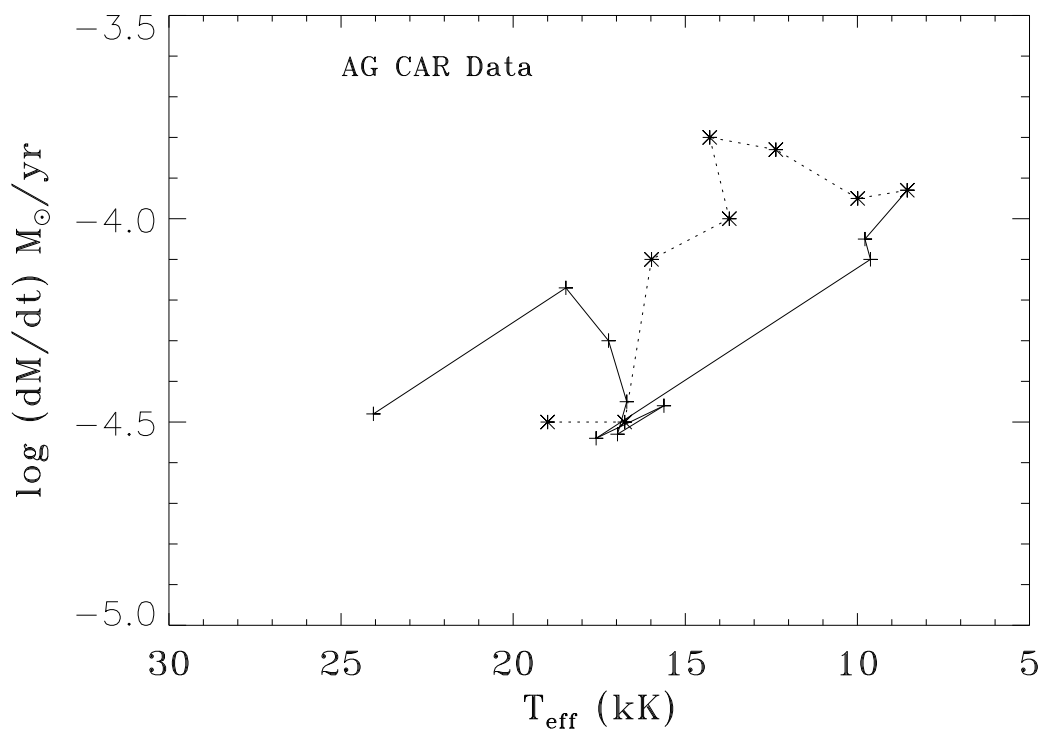

Fig. 3 Time-variable empirical mass-loss rates for AG Car as a function of $T_{\text {eff }}$, as analyzed by Stahl et al. [113]. Figure taken from Vink \& de Koter [127].

is the best studied LBV. Stahl et al. [113] investigated AG Car's mass-loss behavior over the period December 1990 - August 1999 and modelled the $\mathrm{H} \alpha$ profiles in detail. Their empirical mass-loss rates for the cycle from visual minimum to maximum - and back to minimum - are plotted against apparent temperature in Fig. 3 . The mass-loss rate rises, drops, and rises again towards visual maximum (solid line) due to ionization changes of the Fe lines that drive the wind [127]. We note that there is a difference in mass-loss behavior from visual minimum to maximum and in the opposite direction (dotted line). We suspect that this is due to the breakdown of the assumption of stationarity from outburst to quiescence. Due to the larger radii, the dynamical flow times are much longer at maximum than they are at minimum light, which implies that material that was lost in this phase may still be near the photosphere, which may significantly affect the mass-loss determinations, resulting in erroneously large mass-loss rates for the route back to minimum. A second reason for the difference may be related to the release of gravitational energy when the star returns to quiescence. If this plays a role, the assumption of constant bolometric luminosity may no longer hold (see also [36, 13]. Due to the above-mentioned complexities, we focus the comparison of mass-loss predictions to empirical mass-loss rates for the outburst phase (solid line) only. 


\subsection{Theoretical mass-loss rates}

Mass loss from a star with a stationary stellar wind is assumed to be due to an outward acceleration larger than the inward directed gravitational acceleration. For early-type stars, this acceleration has been identified with the radiation force, which depends on both the available photospheric flux and the cross section of the particles that can intercept this radiation.

In hot-star winds, nearly all $\mathrm{H}$ is ionized by the strong radiation field, which implies that there is an enormous number of free electrons present which are the main contributors to the continuum opacity. The radiative acceleration due to photon scattering off free electrons is subject to the same $1 / r^{2}$ radius dependence as is the gravitational acceleration, and for this very reason cannot drive a stellar wind by itself. Lucy \& Solomon [73] showed that a stationary wind would occur when scattering by optically thick spectral lines was included. The interested reader is referred to the introductory book on stellar winds by Lamers \& Cassinelli [62] for an overview of the line acceleration of optically thin and thick lines.

The line acceleration due to all spectral lines is often expressed in terms of the radiative acceleration due to electron scattering times a certain multiplication factor: the force multiplier $M(t)$. Using this method, one can parametrize the line force, and solve the equation of motion in a rather straightforward manner [11]. In this approach, the radiation is assumed to emerge directly from the star. The effects of diffuse radiation and multiple scatterings are not taken into account.

Abbott \& Lucy [1] showed that calculated mass-loss rates can also be obtained using Monte Carlo simulations, counting the cumulative radiative accelerations due to photon interactions with gas particles of different chemical species (mostly Fe). However, the main challenge in radiation-driven wind dynamics is that the line acceleration $g_{\text {line }}$ depends on the velocity gradient $(d V / d r)$, but the velocity $V(r)$, hence $d V / d r$, in turn depends on $g_{\text {line. Due to its non-linear character, the dynamics }}$ of line-driven winds is quite complex. Fortunately, observational analyses provide accurate information on wind velocities, which can be used to constrain the wind dynamics.

Vink \& de Koter [127] adopted an empirical velocity stratification 1 , $V(r)$, and predicted stationary mass-loss rates for LBVs as a function of $T_{\text {eff }}$ in a similar vein to their mass-loss prescriptions for OB supergiants [129]. They studied the effects of lower masses and modified $\mathrm{He} / \mathrm{H}$ and $\mathrm{CNO}$ abundances in comparison to normal $\mathrm{OB}$ supergiants and found that the main difference in mass-loss rate is attributable to the lower masses of LBVs compared to OB supergiants, resulting in a larger Eddington parameter $\Gamma_{\mathrm{e}}$. The increase in He abundance changed the mass-loss properties by only very small amounts (up to about $0.2 \mathrm{dex}$ in $\log \dot{M}$ ). CNO processing also had only a minor effect on the mass-loss rate, because Fe was found to be the dominant contributor to the line force in the inner wind. CNO lines contribute mostly to the line force in the outer wind where the terminal velocity is set [128, 97].

\footnotetext{
${ }^{1}$ Note that our treatment of the winds dynamics has now been updated [82].
} 


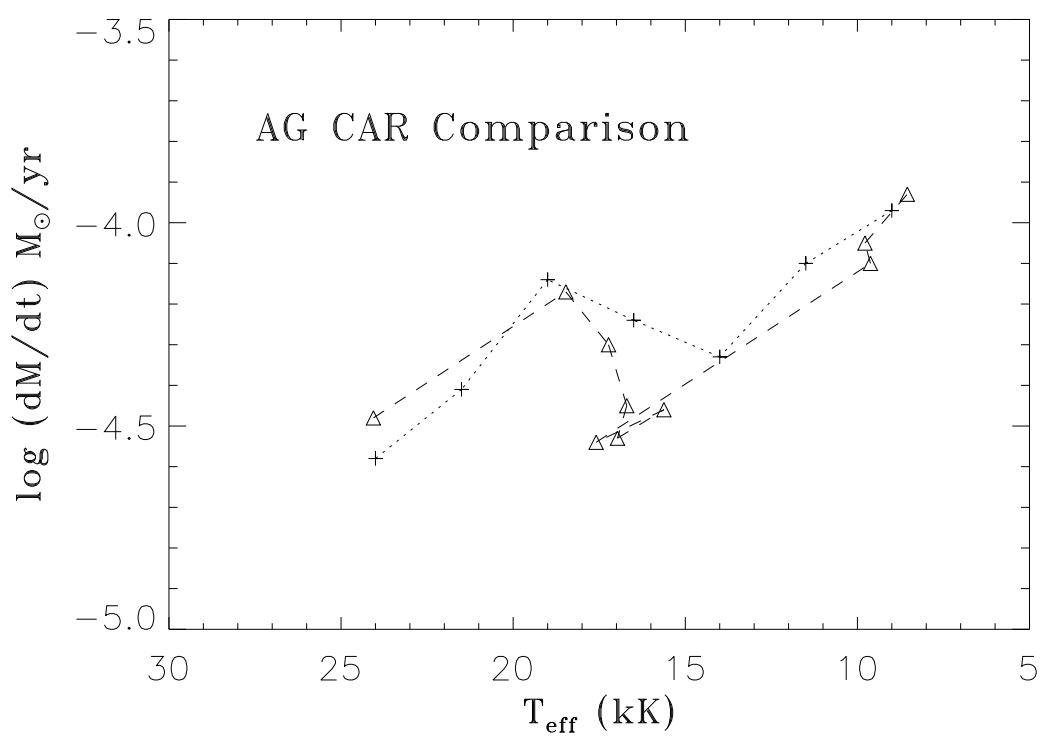

Fig. 4 Predicted (dotted line) and empirical (dashed line) mass-loss rates versus $T_{\text {eff }}$ for the LBV AG Car. Note that both the qualitative behaviour and the amplitude of the mass-loss variations are well reproduced, provided the predictions are shifted by $\Delta T_{\text {eff }}=-6000 \mathrm{~K}$. See [127] for details.

They [127] also compared their LBV mass-loss predictions with observational analyses and showed that the mass-loss variability during the $S$ Dor cycles may arise from changes in the ionization balance of Fe: the bi-stability mechanism, first noticed in model calculations of the wind of P Cygni [93]. The wind either had a high $v_{\infty}$ and low $\dot{M}$, or visa versa. The location of the jump near spectral type B1 (21 000 $\mathrm{K})$ was established by Lamers et al. [64] from a $v_{\infty}$ study of OB supergiants and improved by Crowther et al. [15]. The nature of the jump was originally attributed to the optical thickness of the Lyman continuum [61], but Vink et al. [128] showed that most of the line driving for both the hot and cool side of the jump was due to $\mathrm{Fe}$ in the Balmer continuum, with the jump in mass loss being the result of the recombination of Fe IV, as Fe III has more lines available to drive the wind. The first empirical evidence for a jump in $\dot{M}$ may have been found by Benaglia et al. [5], even though the rates at later spectral types appear to drop below those predicted [129, 118, 76].

The intriguing case of AGCar is depicted in Fig. 4, where we compare the predictions with the Stahl et al. [113] rates when the apparent temperature decreased from 24000 to $9000 \mathrm{~K}$. In these computations, it was assumed that $\log \left(L / L_{\odot}\right)=$ $6.0 ; M=35 M_{\odot}$; the He mass fraction $\mathrm{Y}=0.60$, and the ratio of the terminal over escape velocity was 1.3 . The luminosity and He abundance are similar to those assumed by Stahl et al. We used the mass-loss behavior to constrain the stellar mass of 
the LBV. Unfortunately, the terminal velocity is poorly constrained by observations, as the $v_{\infty}$ determination from $\mathrm{H} \alpha$ only allowed for a lower limit [113]. We note that the adopted ratio of the terminal over the effective escape velocity may impact the mass determination. Furthermore, the Fe recombination temperatures show an offset compared to empirical constraints from the drop in terminal velocities at spectral type B1 in OB supergiants.

Figure 4 shows that after accounting for a corrective shift $\Delta T_{\text {eff }}$, the observed and predicted mass loss agree within $\simeq 0.1 \mathrm{dex}$. As $\dot{M}\left(T_{\text {eff }}\right)$ shows a complex behavior, with fluctuations of over $0.5 \mathrm{dex}$, this is a satisfactory result, confirming that AGCar's mass-loss variability is the result of changes in the ionization of the dominant line-driving element Fe.

\subsection{Do S Dor variables form pseudo-photospheres?}

Now that we have gathered information on the empirical and theoretical mass-loss rates of LBVs, we can start addressing the question of whether these rates are large enough to be capable of forming a pseudo-photosphere. In most modern non-LTE atmosphere codes, the core radius follows from the relation $L=4 \pi R_{\mathrm{in}}^{2} \sigma T_{\mathrm{in}}{ }^{4}$. As the inner boundary is chosen to be deep in the stellar photosphere the input temperature does not necessarily equal the output effective temperature. The effective temperature $T_{\text {eff }}$ is defined at the position where the thermalization optical depth at $5555 \AA$ equals $1 / \sqrt{3}$. We intentionally choose the thermalization optical depth over purely thermal optical depth, as we wish to include the effects of dilution by scattering (see [27] and references therein for more extensive discussions). For stars with modest mass fluxes, such as normal $\mathrm{O}$ stars, the winds are optically thin and $T_{\text {eff }}$ is only slightly lower than $T_{\text {in }}$. For LBVs, with $\dot{M} \sim 10^{-4} M_{\odot} \mathrm{yr}^{-1}$, there may be a significant difference between these temperatures. If the wind is so strong that the optical light originates from the depth of rapid acceleration, the object is considered to be forming a pseudo-photosphere.

The formation of pseudo-photospheres in LBVs may be favored by their lower masses, providing an increased mass-loss rate and an increase of the photospheric scale-height. Leitherer et al. [70] and de Koter et al. [27] assessed whether variable wind properties might explain $\Delta V \simeq 1$ to 2 mags during $\mathrm{S}$ Dor cycles, and concluded that pseudo-photospheres are unlikely to form in LBVs. However, they did not investigate the effect of an order of magnitude change in the wind density of a star that is close to the bi-stability and Eddington limit.

Smith et al. [106] investigated the thermalization optical depth in the inner wind and showed that models on the cool side of the bi-stability jump may start to form optically thick winds which could lead to the formation of a pseudo-photosphere if the objects are close to the Eddington limit. Figure 5 shows that for stars with log $\left(L / L_{\odot}\right)=5.6-5.8$, if the current mass is below $\sim 12 M_{\odot}$, corresponding to $\Gamma_{\mathrm{e}}=0.8$, the star would start to form an extended optically thick wind when it crosses the bi-stability jump, and for stars with masses below $10.5 M_{\odot}\left(\right.$ at $\left.\log \left(L / L_{\odot}\right)=5.7\right)$ 
the pseudo-photosphere starts to form at $T_{\text {eff }} 13000 \mathrm{~K}$. Applying the $\Delta T_{\text {eff }}$ shift of $-6000 \mathrm{~K}$ to match the empirical temperature of the bi-stability jump would bring the effective temperature on the cool side of the bi-stability jump down to $\sim 7000$ $\mathrm{K}$, which agrees with the location of LBVs in eruption and the YHGs.

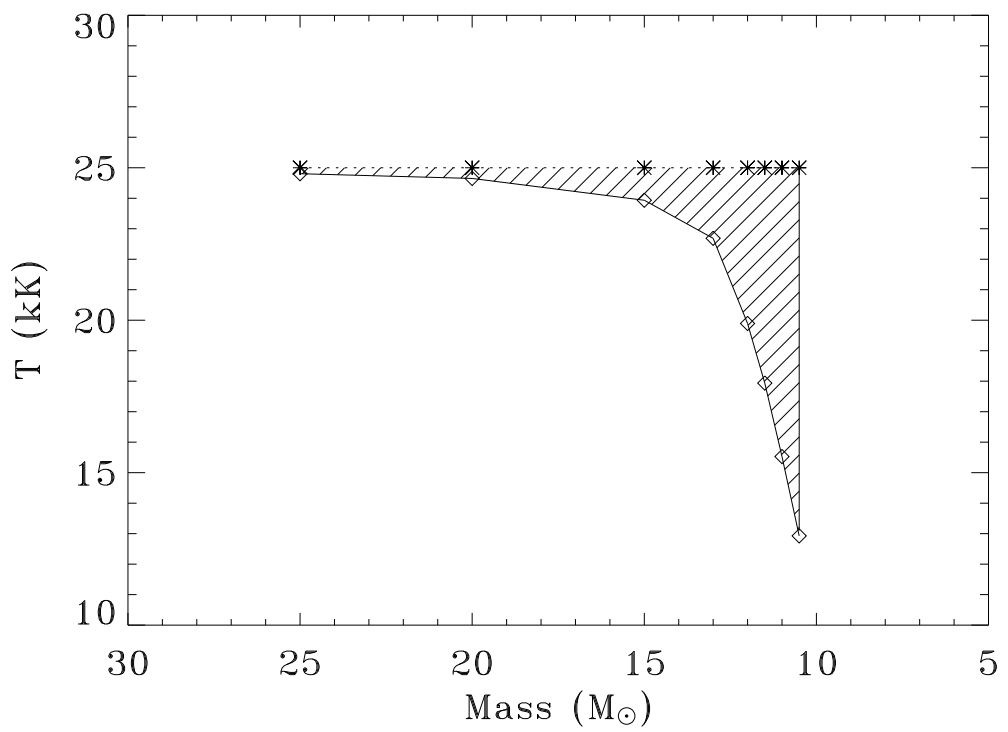

Fig. 5 The possible formation of a pseudo-photosphere. The figure shows the drop in apparent temperature that results from crossing the bi-stability jump which causes an order of magnitude increase in wind density. The apparent temperature drop from $T_{\text {in }}=25000 \mathrm{~K}$ is shown as a function of the stellar mass - for objects with $\log \left(L / L_{\odot}\right)=5.7$. The computed effective output temperatures are denoted with $\diamond$. When the stellar mass drops below $M \sim 10.5 M_{\odot}$, the objects starts to form an extended pseudo-photosphere, which results in an effective temperature of $\sim 13000 \mathrm{~K}$. Applying a corrective shift of $6000 \mathrm{~K}$ [127] this comes down to $T_{\text {eff }} \sim 7000 \mathrm{~K}$ - corresponding to the location of the YHGs. The figure indicates that supergiants that have lost significant mass during prior (e.g. RSG) phases may be susceptible to pseudo-photosphere formation. The figure has been adapted from [106].

The scenario described above hinges critically on the large value of $\Gamma_{\mathrm{e}}$ of $>0.8$, but it remains to be seen if these high $\Gamma_{\mathrm{e}}$ values are realistic for LBVs. We quoted earlier $\Gamma_{\mathrm{e}}$ values in the range $\Gamma_{\mathrm{e}} \simeq 0.5$ for classical, and $\Gamma_{\mathrm{e}} \simeq 0.6$ for low-luminosity LBVs. It is indeed possible that the missing LBVs had larger $\Gamma_{\mathrm{e}}$ values, but clearly, more work is needed to determine LBV masses and luminosities to reliably establish the proximity of LBVs to the Eddington limit. 


\section{Theoretical models for S Dor variability}

In the previous section we addressed the issue of whether the $\mathrm{S}$ Dor variability is the result of a sub-photospheric effect that actually increases the radius resulting in a decrease in $\mathrm{T}_{\text {eff }}$, or whether the apparent decrease in temperature is due to an increase in the mass-loss rate and the formation of an the optically thick wind. It appears that the jury is still out on this, and we should thus consider both atmospheric as well as sub-photospheric mechanisms (see also [50]).

In those cases where a pseudo-photosphere would form as a result of increased mass loss, the root cause of such a sudden $\dot{M}$ increase still needs to be established. The bi-stability mechanism is a good candidate because it can account for observed LBV mass-loss behavior. In the following discussion we include bi-stability under the more general topic of "radiation pressure instabilities".

To investigate which mechanism might be responsible for the large visual brightness and spectroscopic variations in $\mathrm{S}$ Dor variables, we must also consider the momentum balance defined as:

$$
-g_{\text {eff }}=v \frac{d v}{d r}+\frac{1}{\rho} \frac{d P}{d r}
$$

Here $g_{\text {eff }}$ is the net result of gravity, $g_{\mathrm{G}}$, minus the outward-directed radiative and turbulent accelerations $g_{\text {rad }}$ and $g_{\text {turb }}$.

Radiation pressure instability Luminous evolved stars have reduced stability with respect to radiation pressure due to their reduced mass for their luminosity and are thus close to the Eddington limit. However massive stars are also rotating. The critical velocity is defined as $v_{\text {crit }}^{2}=(1-\Gamma) G M / R$. When rotation is included via the $v_{\text {rot }}^{2}$ term in the equation of motion, objects may become unstable when $\Omega=$ $v_{\text {rot }} / v_{\text {crit }}>1$, before arriving at the classical or opacity-modified Eddington limit [66]. For example, the projected rotational velocity for AGCar in the hot phase, of $190 \pm 30 \mathrm{~km} \mathrm{~s}^{-1}$ [35] is close to its critical velocity, and a similar result was recently found for HR Car as well [37]. Thus LBVs are are considered to be in close proximity to both the Eddington and the Omega limits. In the sense usually intended, the $\Omega$ limit is really the Eddington limit modified by rotation 2 Thus, in the following we include the Omega $(\Omega)$ limit under the general topic of the "Eddington limit".

Radiation-pressure driven instabilities occur because as the temperature drops, the opacity rises (e.g. due to bi-stability), and the radiative acceleration $g_{\text {rad }}$ increases. The opacity-modified Eddington limit was initially introduced to explain the great eruption of $\eta$ Car [17], and subsequently for the temperature dependence of the HD limit [49, 59], and the instability of LBVs [3, 4]. Lamers \& Fitzpatrick [60] computed the location of the opacity-modified Eddington limit, including metal-line

\footnotetext{
${ }^{2}$ In principle this is a simplification of the real physics, because rotation tends to make the equator cooler than the poles (von Zeipel effect). The resulting temperature gradient affects opacity and possible instabilities, potentially making the combination of radiation pressure and rotation synergistic as noted in [61, 94] as well as section 5 of [138
} 
opacities from model atmospheres in addition to electron scattering. They suggested that S Dor variations could result from a conflict between a star's tendency to expand (following core H-burning) and strong mass loss close to the Eddington limit, requiring the star to shrink as the mass decreases. However there are issues with this simplified approach. When the ratio of radiative to gravitational force approaches unity, an instability could be expected, but as the atmosphere expands and density decreases, the ionization increases thereby reducing the absorptive opacity, and instead it approaches the classical Eddington limit due to electron scattering (which is not temperature dependent). However, if the instability would occur at $\Gamma$ somewhat less than unity, the density decrease would not eliminate absorption, and the concept of the modified Eddington limit might nonetheless work.

The attraction of scenarios based on the Eddington limit are clear; they naturally explain the temperature dependent luminosity limit in the HRD, the S Dor variability, and the two states of LBVs, their high $T_{\text {eff }}$ (just on the hot side of the bi-stability jump), and their low $T_{\text {eff }}$ limits. They could also lead to enhanced mass loss, increased density in the winds and the formation of a pseudo-photosphere. However, there are no self-consistent models that provide a sound theoretical basis for for scenarios involving pseudo-photospheres [27].

Turbulent pressure instability As a star approaches the Eddington limit, the outermost layers of the envelope become convective (e.g. [10]) and turbulent pressure gradients may provide an additional acceleration, $g_{\text {turb }}$ to the momentum equation. De Jager [25] showed how supersonic turbulence may destabilize the atmosphere, and as the mechanism becomes more efficient at higher luminosity, the mass-loss rate increases. This is also true for radiation pressure forces and it may be difficult to distinguish between these two atmospheric instabilities.

\section{Vibrations and dynamical instability}

Together with the radiation pressure-based instabilities, sub-photospheric dynamical mechanisms are the most promising explanation for the LBV/S Dor variability (see Gräfener et al. 2011b and references therein). In these models, "strange modes" and dynamical instabilities are caused by the bump or increase in the opacity due to iron at the base of the photosphere leading to a strong ionization-induced instability in the outer envelope as stars transit the HRD after the end of core H-burning. In the models of Stothers \& Chin [117] the star keeps re-adjusting itself on thermal timescales after periods of strong mass loss, whilst shrinking in radius. These models provide the correct S Dor timescales and also appear to "behave" properly at constant bolometric luminosity. The strange mode calculations by Glatzel and Kiriakidis [33] reproduce the the S Dor instability strip and the upper luminosity boundary in the HRD quite well. Dynamical instability thus remains one of the more promising candidates to explain LBV variability.

Vibrational or pulsational instability was once thought to be one of the main contenders for instability and mass loss in the most massive stars (e.g. [2]), but the $\mathcal{E}$-mechanism is energized in the core, appears to grow too slowly (e.g. [88]) and is therefore no longer considered valid for LBVs. Another sub-photospheric instabil- 
ity, the $\kappa$ mechanism responsible for pulsation in massive stars such as the Cepheids may also cause pulsations in the outer envelope. It may be responsible for some of the micro-variability seen in LBVs and other supergiants ([68] and references therein) which occur on timescales of weeks to months. The timescale of the S Dor variations however is much longer and therefore unlikely to be due to pulsations.

Binarity Most LBVs are apparently single, and although. $\eta$ Car may have a companion, it seems clear that binarity can neither be the root cause of the S Dor variations, or for the giant eruptions, as the only other local example, P Cyg, is single (but see [57], or unless it formed through merging, cf. [95]).

\section{Evolutionary State}

There is no doubt that LBVs are evolved, unstable massive hot stars. The more massive classical LBVs have apparently evolved off the main sequence, while the less luminous LBVs may be post-red supergiants. In the generally accepted view of massive star evolution, the classical LBVs are considered "transitional" objects in a phase before entering the He-burning WR stage [67], by the end of which the star is anticipated to explode as a type Ib/c supernova. Many LBVs are known to be $\mathrm{N}$ and $\mathrm{He}$ rich compared to $\mathrm{O}$ stars, but $\mathrm{H}$ rich compared to the more evolved WR stars. This situation is somewhat more complex as there is also a group of highluminosity late-type H-rich WR stars, which appear closely related to many classical LBVs in quiescence the Ofpe/WN stars [131]. These and other luminous stars, the $\mathrm{B}$ [e] supergiants [65], and and the cool or yellow Hypergiants (YHGs) [26], that show evidence for high mass loss and instabilities which may be related to the LBV state.

\subsection{The evolutionary neighbours}

Ofpe/late-WN stars These "slash" stars are a group of luminous hot stars with very strong emission lines due to their strong mass loss. They have He and $\mathrm{N}$ enhanced atmospheres indicative of an evolved state [89], but are generally not believed to be highly variable. These stars however may be closely related to the LBVs in quiescence. The S Dor-type variable, R127, was a late-WN star prior to its long-term outburst beginning in the early 1980s [111]. Either the late-WN stars evolve into LBVs, or they may represent a dormant phase of LBV evolution. Either way, Ofpe/late-WN stars are thought to be evolved massive stars in a transitional stage for objects with initial masses $M>50-60 M_{\odot}$. Since no evolved stars are observed redwards of the Humphreys-Davidson (HD) limit at these high luminosities and masses, high mass loss during the Ofpe/late-WN and LBV phases may reverse the evolutionary track back to the hotter part of the HRD, where they should appear as He-burning WR 
stars.

$\mathbf{B}[\mathbf{e}]$ supergiants The spectra of the $\mathrm{B}[\mathrm{e}]$ stars [65] show an abundance of highexcitation permitted and forbidden emission lines that are thought to arise from an equatorially enhanced outflowing disk. Zickgraf et al. [139] proposed a 2component wind with a normal fast polar wind and a dense slow outflowing equatorial "disk". A popular mechanism to explain this 2-component wind is the rotationally induced bi-stability mechanism [61, 94]. The pole is hotter than the equator, due to the Von Zeipel gravity darkening effect which could lead to a fast, low $\dot{M}$, polar wind driven by Fe IV lines, and a slow, high $\dot{M}$, equatorial wind driven by the more effective Fe III lines [128]. This mechanism is expected to occur predominantly at spectral type B. However the star is expected to rotate rapidly but $v_{\text {rot }}$ measurements are difficult for $\mathrm{B}[\mathrm{e}]$ stars, with most of the lines in emission.

The $\mathrm{B}[\mathrm{e}]$ supergiants may represent a subset of massive stars with high rotational velocities. The $\mathrm{B}[\mathrm{e}]$ supergiants were originally not thought to be variable, but there is now evidence for large amplitude variability for some $\mathrm{B}[\mathrm{e}]$ supergiants [124], suggesting a closer evolutionary link between LBVs and $\mathrm{B}[\mathrm{e}]$ supergiants than previously acknowledged.

Yellow or cool hypergiants YHGs are found just below the HD-limit [48, 50] at intermediate temperatures with A to G spectral types. Many of these stars show spectroscopic and photometric variability, high mass-loss rates, large infrared excesses and visible circumstellar ejecta, all evidence for instability. The YHGs are often assumed to be post-RSGs [26, 87], although the evolutionary state is not established for all of them. The intriguing object IRC +10420 has been shown to be a post-RSG [56, 86] and numerous studies have revealed its complex ejecta [51, 53] and large-scale asymmetry [92, 28, 23]. Given their variability and high mass loss, the YHGs are likely close to their Eddington limit, with a large $\Gamma_{e}$, and it is thus probable that many of them are post-RSGs Nevertheless, it is not clear whether objects like IRC +10420 are on an evolutionary blueward journey towards the WR phase [86, 53] bouncing against the yellow void [26], or are on the cool side of the bi-stability jump [106].

\subsection{Do LBVs explode?}

The picture of the LBVs as a high mass loss, relatively short-lived (some $10^{4} \mathrm{yrs}$ ) and presumably core H-burning phase prior to a much longer (a few times $10^{5} \mathrm{yrs}$ ) core He-burning WR phase seemed well established - until recently.

There is increasing observational evidence that LBVs could be direct progenitors of some SNe. Kotak \& Vink [58] proposed that the quasi-periodic modulations seen in the radio light curves of transitional SNe such as SN 2001ig and SN 2003bg are the manifestation of variable mass loss during S Dor excursions. Although several other possibilities have been put forward to explain these modulations [99, 109], 
none has been entirely satisfactory. The recurrence timescale of the variability, as well as the amplitude of the radio modulations are consistent with those of S Dor variables and their scenario [58] provides a rather natural explanation for a behaviour that is expected on theoretical grounds [127].

The same wind bi-stability mechanism may be able to account for wind-velocity variations seen spectroscopically in SN 2005gj [119] in which the variable winds are inferred from double P Cygni components (see Fig.6) which appear almost identical to those seen in the $\mathrm{H} \alpha$ profiles of S Dor variables like AG Car and HD 160529 (see also [34]). Both the timescales and the spectroscopically measured wind velocities of SN 2005gj, with $v_{\infty} \simeq 100-200 \mathrm{~km} \mathrm{~s}^{-1}$, are consistent with those of LBVs, but not with those of the much slower RSG winds $\left(\sim 10 \mathrm{~km} \mathrm{~s}^{-1}\right)$, or the much faster WR winds $\left(\simeq 1000-5000 \mathrm{~km} \mathrm{~s}^{-1}\right)$. See Van Marle et al. [125] and [119] for more information.

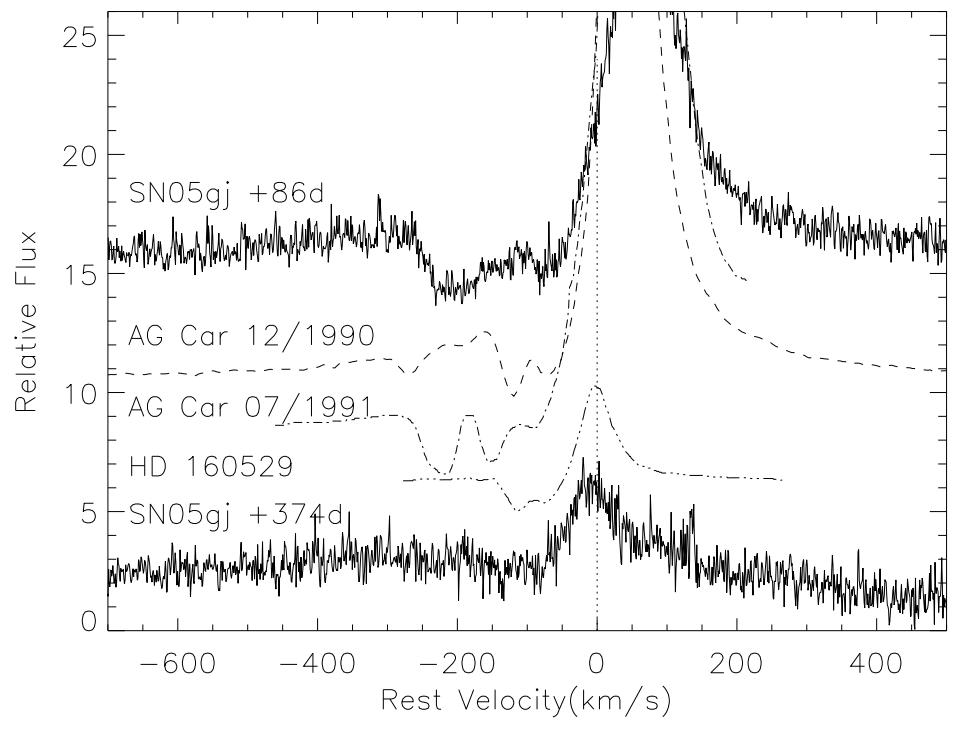

Fig. 6 Multiple absorption components seen in the P Cygni $\mathrm{H} \alpha$ profile of SN 2005gj (top) in comparison to the LBVs AG Car and HD 160529. The figure has been taken from Trundle et al. [119.

The progenitor star of the recent supernova SN 2006jc had a giant eruption just two years before its terminal explosion. Foley et al. [30] and Pastorello et al. [91] suggested that the progenitor star was either a WR star that exhibited an "LBV or $\eta$ Car-like" eruption, or that the progenitor was part of a binary system including both an LBV and a WR star, with the WR star exploding and the giant eruption attributable to the LBV, as WR stars have never been observed to have an $\eta$ Car type eruptions. A more direct application of Occam's razor would be to accept that 
the progenitor object exploded during or at the end of the LBV phase or an $\eta$ Carlike giant eruption [58].

Gal-Yam et al. [31] discovered a luminous source (with $M_{\mathrm{bol}}=-10.3$ ) in the pre-explosion image of SN 2005gl. Although, the properties of the progenitor are consistent with those of LBVs, they are equally consistent with a luminous blue supergiant that has not exhibited spectral type variations (see the membership discussion in Sect. 21, and the progenitor of SN 2005gl may potentially be classified as an LBVc. The resulting SN explosion was of type IIn, indicating the presence of a dense circumstellar medium, additional evidence for prior $\eta$ Car-type eruptions.

Other hints that LBVs may explode come from similarities in the morphology of LBV nebula and the circumstellar medium of SN 1987A [103], while the very luminous SN 2006gy - hypothesized to be an exotic pair-instability SN - may also have undergone an $\eta$ Car-type eruption before exploding [107]. An alternative scenario for a giant $\eta$ Car-type outburst was suggested by Woosley et al. [136] who attributed the dense shells around this luminous SN to pulsational pair instability.

We emphasize that the evolutionary status of LBVs remains uncertain (see the discussion in [29]). Evolutionary models have been constructed to allow LBVs in a transitional phase between a core H-burning main sequence and a core He-burning WR phase. This model naturally accounts for the the chemical abundances $(\mathrm{He}, \mathrm{N})$ of LBVs which are intermediate between those of $\mathrm{O}$ and WR stars. The concept of an LBV exploding is certainly at odds with current stellar evolution models and an exploding LBV scenario was until recently considered "wildly speculative" [104]. Nevertheless, the evolutionary models do not provide a straightforward explanation for the wide range of phenomena described above and a simple explanation for this could be that at least some massive stars in an LBV state could precede the terminal explosion. This would allow some massive stars to skip the WR phase - in contradiction to the basic framework of massive star evolution. This also suggests that LBVs are already in the core He-burning phase of evolution. Given the intriguing variations seen in radio lightcurves and especially the double absorptions seen spectroscopically in PCygni line profiles, I suggest that the changing winds of LBVs may help addressing the issue of whether the LBV phase may indeed represent the evolutionary endpoint for some of the most massive stars.

\section{Outlook}

We have examined observational, atmospheric modelling, and theoretical aspects of the current status of our knowledge of the LBV instability and their role in massive star evolution. Although radiation pressure as well as a dynamical instability are strong candidates for explaining S Dor variability, we conclude that the mechanism at the origin of the LBV phenomenon remains elusive. One of the most relevant issues to be addressed relates to the nature of the $\mathrm{S}$ Dor variability itself. With respect to massive star evolution, and in particular whether LBVs are in a transitional or final 
phase of evolution, the last few years have seen a flurry of activity, but we should not yet draw any definitive conclusions regarding their evolutionary state.

Progress is expected to be made on a number of fronts. First of all, the number of known LBVs is small and for a proper understanding of any individual member, photometric, polarimetric, and spectroscopic monitoring on the timescale of the $\mathrm{S}$ Dor variations is required. Atmospheric modelling is necessary to determine the stellar parameters and place the LBVs properly onto the HRD. There are still enormous uncertainties in the values shown in Fig. 11 Furthermore, LBV masses, nor their proximity to the Eddington limit, are known with any level of certainty, with profound consequences for theoretical interpretation. Therefore, binary searches, orbit determinations, and spectroscopic modelling are strongly encouraged to determine this most basic parameter.

As is the case for the normal OB supergiants, the LBV mass-loss rates are uncertain due to wind clumping. Progress will undoubtedly be made regarding the role of of wind clumping and its impact on mass-loss rates in OB stars and LBVs. However, the LBVs themselves provide an ideal laboratory for studies of wind clumping, because the polarization variability is most extreme, due to the combined effect of low outflow velocities and high mass-loss rates [21]. Mass-loss variability - in conjunction with $T_{\text {eff }}$ determinations - could also be utilized to constrain the stellar masses, as was exemplified for the object AG Car [127].

A number of developments provide new opportunities for LBV research. The infrared era has properly come of age with satellites such as Spitzer. Recent activities involve both in-depth studies of known LBVs [120], as well as the discoveries of new ones, even beyond the local group. We highlight the so-called intermediate luminosity transients, where "intermediate" means that their luminosities are in between classical novae and supernovae. The nature of these transients, incl. SN 2008S and NGC 300 OT2008-1, remains as yet elusive. Some authors argue for a SN origin [96, 8], whilst others [6, 7, 108] suggest they are due to the non-terminal outburst of an LBV-type star. Another intriguing direction for LBV research is provided by the detection of LBVs and candidates in very low metallicity environments [98, 55, 43]. The mere presence of LBVs at such low metallicities presents a challenge for theory, because of the reduced opacity available to drive an LBV wind.

Further theoretical work on the formation of pseudo-photospheres and the more general question regarding the origin of the LBV variations are badly needed. Such studies may result in a better understanding of the origin of the variability which is necessary to place the LBV phase correctly in the evolution of massive stars.

The final message to emerge from this chapter is that $\eta$ Car may be one of the most extreme LBVs but it is not unique among the LBVs. Given that $\eta$ Car's second outburst (during 1888-1895) was like that of a normal S Dor variable, the key to our understanding of $\eta$ Car's great eruption may thus not exclusively lie in the study of $\eta$ Car itself, but pivotal clues may be obtained through a better understanding of the more typical S Dor variations that define the LBVs as a class among the most massive stars.

The most outstanding question for all these stars, S Dor-type LBVs and the giant eruptions like $\eta$ Car, is still, what is the underlying origin of their instabilities? 
Acknowledgements I am grateful for the many discussions I have had with colleagues from the massive star community and in particular to Alex de Koter, Henny Lamers, and Rubina Kotak.

\section{References}

1. Abbott, D.C., Lucy L.B.: Multiline transfer and the dynamics of stellar winds, ApJ 288, 679693 (1985)

2. Appenzeller, I.: Mass Loss Rates for Vibrationally Unstable Very Massive Main-sequence Stars, A\&A 9, 216-220 (1970)

3. Appenzeller, I.: Instability in massive stars - an overview, IAUS 116, 139-148 (1986)

4. Appenzeller, I.: The Role of Radiation Pressure in LBV Atmospheres, IAUC, 113, 195-202 (1989)

5. Benaglia, P., Vink, J.S., Marti, J., Maiz Apellaniz, J., Koribalski, B., Crowther, P.A.: Testing the predicted mass-loss bi-stability jump at radio wavelengths, A\&A 467, 1265-1274 (2007)

6. Berger, E., Soderberg, A.M., Chevalier, R.A., et al.: An Intermediate Luminosity Transient in NGC 300: The Eruption of a Dust-Enshrouded Massive Star, ApJ 699, 1850 (2009)

7. Bond, H.E., Bedin, L.R., Bonanos, A.Z., et al.: The 2008 Luminous Optical Transient in the Nearby Galaxy NGC 300, ApJ 695, 154 (2009)

8. Botticella, M.T., Pastorello, A., Smartt, S.J., et al.: SN 2008S: an electron-capture SN from a super-AGB progenitor?, MNRAS 398, 1041 (2009)

9. Buemi, C., Distefano, E., Leto, P., et al.: Photometric monitoring of Luminous Blue Variables, BSRSL 80, 335 (2011)

10. Cantiello, M., Langer, N., Brott, I., et al.: Sub-surface convection zones in hot massive stars and their observable consequences, A\&A 499, 279-290 (2009)

11. Castor J.I., Abbott D.C., Klein R.I.: Radiation-driven winds in Of stars, ApJ 195, 157-174 (1975)

12. Clark, J.S., Larionov, V.M., Arkharov, A.: On the population of galactic Luminous Blue Variables, A\&A 435, 239-246 (2005)

13. Clark, J.S., Crowther, P.A., Larionov, et al.: Bolometric luminosity variations in the luminous blue variable AFGL2298, A\&A 507, 1555-1565 (2009)

14. Cox, P., Mezger, P.G., Sievers, A., et al.: Millimeter emission of eta Carinae and its surroundings, A\&A 297, 168-174 (1995)

15. Crowther, P.A., Lennon, D.J., Walborn, N.R.: Physical parameters and wind properties of galactic early B supergiants, A\&A, 446, 279-293 (2006)

16. Damineli, A.: The 5.52 Year Cycle of Eta Carinae, ApJ 460, L49-L52 (1996)

17. Davidson, K.: On the nature of Eta Carinae, MNRAS 154, 415-427 (1971)

18. Davidson, K.: The relation between apparent temperature and mass-loss rate in hypergiant eruptions, ApJ 317, 760-764 (1987)

19. Davidson, K., Walborn, N.R., Gull, T.R.: The remarkable spectrum of some material ejected by Eta Carinae, ApJ 254, L47-L51 (1982)

20. Davidson, K., Ebbets, D., Weigelt, G.: HST/FOS spectroscopy of ETA Carinae: the star itself, and ejecta within 0.3 arcsec, AJ 109, 1784-1796 (1995)

21. Davies B., Oudmaijer R.D., Vink J.S.: Asphericity and clumpiness in the winds of Luminous Blue Variables, A\&A 439, 1107-1125 (2005)

22. Davies, B., Vink, J.S., Oudmaijer, R.D.: Modelling the clumping-induced polarimetric variability of hot star winds, A\&A 469, 1045-1056 (2007)

23. Davies, B., Oudmaijer, R.D.; Sahu, K.C.: Integral-Field Spectroscopy of the Post-Red Supergiant IRC +10420: Evidence for an Axisymmetric Wind, ApJ 671, 2059-2067 (2007)

24. de Groot, M., Sterken, C., van Genderen, A.M.: Cyclicities in the light variations of S Doradus stars III. P Cygni, A\&A 376, 224-231 (2001)

25. de Jager, C.: The stability limit of hypergiant photospheres, A\&A 138, 246-252 (1984) 
26. de Jager, C.: The yellow hypergiants, A\&ARv 8, 145-180 (1998)

27. de Koter, A., Lamers, H.J.G.L.M., Schmutz, W.: Variability of luminous blue variables. II. Parameter study of the typical LBV variations, A\&A 306, 501-518 (1996)

28. de Wit, W.J., Oudmaijer, R.D., Groenewegen, M.A.T., Hoare, M.G., Malbet, F.: Resolving the ionized wind of the post-red supergiant IRC +10 420 with VLTI/AMBER, A\&A 480, 149-155 (2008)

29. Dwarkadas, V.V.: On luminous blue variables as the progenitors of core-collapse supernovae, especially Type IIn supernovae, MNRAS 412, 1639-1649 (2011)

30. Foley, R.J., Smith, N., Ganeshalingam, M., et al.: SN 2006jc: A Wolf-Rayet Star Exploding in a Dense He-rich Circumstellar Medium, ApJ 657, L105-L108 (2007)

31. Gal-Yam, A., Leonard, D.C., Fox, D.B., et al.: On the Progenitor of SN $2005 \mathrm{gl}$ and the Nature of Type IIn Supernovae, ApJ, 656, 372-381 (2007)

32. Garcia-Segura, G., Langer, N., Mac Low, M.-M.: The dynamical evolution of circumstellar gas around massive stars. I. The impact of the time sequence Ostar - LBV - WR star, A\&A 305, 229-244 (1996)

33. Glatzel, W., Kiriakidis, M.: Stability of Massive Stars and the Humphreys-Davidson Limit, MNRAS 263, 375-384 (1993)

34. Groh, J.H., Vink, J.S.: The bi-stability jump as the origin for multiple P-Cygni absorption components in luminous blue variables, A\&AL 513, 10-13 (2011)

35. Groh, J.H., Hillier, D.J., Damineli, A.: AG Carinae: A Luminous Blue Variable with a High Rotational Velocity, ApJ 638, L33-L36 (2006)

36. Groh, J.H., Hillier, D.J., Damineli, A., Whitelock, P.A., Marang, F., Rossi, C.: On the Nature of the Prototype Luminous Blue Variable AG Carinae. I. Fundamental Parameters During Visual Minimum Phases and Changes in the Bolometric Luminosity During the S-Dor Cycle, ApJ 698, 1698-1720 (2009)

37. Groh, J.H., Hillier, D.J., Damineli, A., et al.: Bona Fide, Strong-Variable Galactic Luminous Blue Variable Stars are Fast Rotators: Detection of a High Rotational Velocity in HR Carinae, ApJL 705, L25-L30 (2009)

38. Guo, J.H., Li, Y.: Estimating the Structure and Geometry of Winds from Luminous Blue Variables via Fitting the Continuum Energy Distributions, ApJ 659, 1563-1575 (2007)

39. Hamann, W.-R., Feldmeier, A., Oskinova, L.M., 2008, Clumping in hot-star winds: proceedings of an international workshop held in Potsdam, Germany, 18. - 22. June 2007 (2008)

40. Gvaramadze, V.V., Kniazev, A.Y., Fabrika, S., et al.: MN112: a new Galactic candidate luminous blue variable, MNRAS 408 (2010)

41. Harries T.J., Hillier D.J., Howarth I.D.: A spectropolarimetric survey of northern hemisphere Wolf-Rayet stars, MNRAS 296, 1072-1088 (1998)

42. Harries, T.J., Howarth, I.D., Evans, C.J.: Spectropolarimetry of O supergiants, MNRAS 337, 341-355 (2002)

43. Herrero, A., Garcia, M., Uytterhoeven, K., et al.: The nature of V39: an LBV candidate or LBV impostor in the very low metallicity galaxy IC 1613?, \&A 513, 70 (2010)

44. Hillier, D.J., Miller, D.L.: The Treatment of Non-LTE Line Blanketing in Spherically Expanding Outflows, ApJ 496, 407 (1998)

45. Hillier, D.J., Crowther, P.A., Najarro, F., Fullerton, A.W.: An optical and near-IR spectroscopic study of the extreme P Cygni-type supergiant HDE 316285, A\&A 340, 483-496 (1998)

46. Hillier, D.J., Davidson, K., Ishibashi, K., Gull, T.: On the Nature of the Central Source in $\eta$ Carinae, ApJ 553, 837-860 (2001)

47. Hubble, E., Sandage, A.: The Brightest Variable Stars in Extragalactic Nebulae. I. M31 and M33, ApJ 118, 353-361 (1953)

48. Humphreys, R.M., Davidson, K.: Studies of luminous stars in nearby galaxies. III - Comments on the evolution of the most massive stars in the Milky Way and the Large Magellanic Cloud, ApJ 232, 409-420 (1979)

49. Humphreys, R.M., Davidson, K.: The Most Luminous Stars, Science 223, 243-249 (1984)

50. Humphreys, R.M., Davidson, K.: The luminous blue variables: Astrophysical geysers, PASP 106, 1025-1051 (1994) 
51. Humphreys, R.M., Smith, N., Davidson, et al.: HST and Infrared Images of the Circumstellar Environment of the Cool Hypergiant IRC + 10420, AJ 114, 2778 (1997)

52. Humphreys, R.M., Davidson, K., Smith, N.: Eta Carinae's Second Eruption and the Light Curves of the eta Carinae Variables, PASP 111, 1124-1131 (1999)

53. Humphreys, R.M., Davidson, K., Smith, N.: Crossing the Yellow Void: Spatially Resolved Spectroscopy of the Post-Red Supergiant IRC +10420 and Its Circumstellar Ejecta, AJ 124, 1026-1044 (2002)

54. Hutchings, J. B.,:Stellar Winds from Hot Supergiants, ApJ 203, 438 (1976)

55. Izotov, Y.I., Thuan, T.X.: Luminous Blue Variable Stars in the two Extremely Metal-Deficient Blue Compact Dwarf Galaxies DDO 68 and PHL 293B, ApJ 690, 1797-1806 (2009)

56. Jones, T.J., Humphreys, R.M., Gehrz, R.D., et al.: IRC +10420 - A cool hypergiant near the top of the H-R diagram, ApJ 411, 323-335 (1993)

57. Kashi, A.: An Indication for the Binarity of P Cygni from Its Seventeenth Century Eruption, MNRAS in press, http://arxiv.org/abs/0912.3998 (2010)

58. Kotak, R. Vink, J.S.: Luminous blue variables as the progenitors of supernovae with quasiperiodic radio modulations, A\&A, 460, L5-L8 (2006)

59. Lamers, H.J.G.L.M.: P Cygni type stars - Evolution and physical processes, IAUS 116, 157$178(1986)$

60. Lamers, H.J.G.L.M., Fitzpatrick, E.L.: The relationship between the Eddington limit, the observed upper luminosity limit for massive stars, and the luminous blue variables, ApJ 324, 279-287 (1988)

61. Lamers, H.J.G.L.M., Pauldrach, A.W.A.: The formation of outflowing disks around earlytype stars by bi-stable radiation-driven winds, A\&A 244, L5-L8 (1991)

62. Lamers, H.J.G.L.M., Cassinelli, J.P.: Introduction to Stellar Winds (Cambridge University Press) (1999)

63. Lamers, H.J.G.L.M., Korevaar, P., Cassatella, A.: The ejection of shells in the stellar wind of the hypergiant P Cygni (B1 Ia/+/), A\&A 149, 29-40 (1985)

64. Lamers, H.J.G.L.M., Snow, Th. P., Lindholm, D.M.: Terminal Velocities and the Bistability of Stellar Winds, ApJ 455, 269 (1995)

65. Lamers, H.J.G.L.M., Zickgraf, F.-J.; de Winter, D., Houziaux, L., Zorec, J.: An improved classification of B[e]-type stars, A\&A 340, 117-128 (1998)

66. Langer, N.: The Eddington Limit in Rotating Massive Stars, ASPC 120, 83 (1997)

67. Langer, N., Hamann, W-R., Lennon, M., et al.: Towards an understanding of very massive stars. A new evolutionary scenario relating O stars, LBVs and Wolf-Rayet stars, A\&A 290, 819-833 (1994)

68. Lefever, K., Puls, J., Aerts, C.: Statistical properties of a sample of periodically variable Btype supergiants. Evidence for opacity-driven gravity-mode oscillations, A\&A 463, 10931109 (2007)

69. Leitherer, C.: Mass Loss from LBVs: Observational Constraints, ASPC 120, $58-65$ (1997)

70. Leitherer, C., Schmutz, W., Abbott, D.C., Hamann, W.-R, Wessolowski, U.: Atmospheric models for luminous blue variables, ApJ 346, 919-931 (1989)

71. Lennon, D.J., Wobig, D., Kudritzki, R.-P., Stahl, O.: The atmospheric composition, extinction and luminosity of the LBV star R71, SSRv 66, 207 (1993)

72. Lucy L.B.: An analysis of the variable radial velocity of alpha Cygni, ApJ 206, 499-508 (1976)

73. Lucy L.B., Solomon P.M.: Mass Loss by Hot Stars, ApJ 159, 879-893 (1970)

74. Maeder, A.: Evolution of chemical abundances in massive stars. I - OB stars, Hubble-Sandage variables and Wolf-Rayet stars - Changes at stellar surfaces and galactic enrichment by stellar winds. II - Abundance anomalies in Wolf-Rayet stars in relation with cosmic rays and 22/Ne in meteorites, A\&A 120, 113-135 (1983)

75. Maryeva, O., Abolmasov, P.: Modeling the optical spectrum of Romano's star, arXiv: 1109.0443 (2011)

76. Markova, N., Puls, J.: Bright OB stars in the Galaxy. IV. Stellar and wind parameters of early to late B supergiants, A\&A 478, 823-842 (2008) 
77. Markova, N., Morrison, N., Kolka, I., Markov, H.: P Cygni in a short S Doradus phase. Spectroscopic and photometric evidences, A\&A 376, 898-906 (2001)

78. Martins, F., Trippe, S., Paumard, T., et al.: GCIRS 16SW: A Massive Eclipsing Binary in the Galactic Center, ApJ 649, L103-L106 (2006)

79. Massey, P., McNeill, R.T., Olsen, K.A.G., et al.: A Survey of Local Group Galaxies Currently Forming Stars. III. A Search for Luminous Blue Variables and Other H Emission-Line Stars, AJ 134, 2474-2503 (2007)

80. Mauerhan, J.C., Morris, M.R., Cotera, A., et al.: Discovery of a Luminous Blue Variable with an Ejection Nebula Near the Quintuplet Cluster, ApJ 713, 33 (2010)

81. Meynet G. Maeder A.: Stellar evolution with rotation. XI. Wolf-Rayet star populations at different metallicities, A\&A 429, 581-598 (2005)

82. Müller, P.E., Vink, J.S.: A consistent solution for the velocity field and mass-loss rate of massive stars, A\&A 492, 493-509 (2008)

83. Najarro, F., Hillier, D.J., Stahl, O.: A spectroscopic investigation of P Cygni. I. H and HeI lines, A\&A 326, 1117-1134 (1997)

84. Nordsieck K.H., Wisniewski J., Babler B.L, et al.: Ultraviolet and visible spectropolarimetric variability in P Cygni, ASPC 233, 261-274 (2001)

85. Nota, A. Livio, M., Clampin, M., Schulte-Ladbeck, R.: Nebulae around Luminous Blue Variables: A Unified Picture, ApJ 448, 788-796 (1995)

86. Oudmaijer, R.D., Groenewegen, M.A.T., Matthews, H.E., Blommaert, J.A.D.L., Sahu, K.C.: The spectral energy distribution and mass-loss history of IRC+10420, MNRAS 280, 1062-70 (1996)

87. Oudmaijer, R.D., Davies, B., de Wit, W.-J.; Patel, M.: Post-Red Supergiants, astro$\mathrm{ph} / 0801.2315$ (2008)

88. Papaloizou, J.C.B.: Non-linear pulsations of upper main sequence stars-II.Direct numerical integrations, MNRAS 162, 169 (1973)

89. Pasquali, A., Langer, N., Schmutz, W.: O Stars in Transition. II. Fundamental Properties and Evolutionary Status of Ofpe/WN9 Stars from Hubble Space Telescope Ultraviolet Observations, ApJ 478, 340-357 (1997)

90. Pasquali, A., Nota,A., Smith, L.J., et al.: Multiwavelength Study of the Nebula Associated with the Galactic LBV Candidate HD 168625, AJ 124, 1625-1635 (2002)

91. Pastorello, A., Smartt, S. J., Mattila, S., et al.: A giant outburst two years before the corecollapse of a massive star, Nature 447, 7146, 829-832 (2007)

92. Patel, M., Oudmaijer, R.D., Vink, J.S., et al.: Spectropolarimetry of the massive post-red supergiants IRC +10420 and HD 179821, MNRAS 385, 967-978 (2008)

93. Pauldrach, A.W.A., Puls, J.: Radiation-driven winds of hot stars. VIII - The bistable wind of the luminous blue variable P Cygni (B1 Ia/+/), A\&A, 237, 409-424 (1990)

94. Pelupessy I., Lamers H.J.G.L.M., Vink J.S.: The radiation driven winds of rotating B[e] supergiants, A\&A 359, 695-706 (2000)

95. Podsiadlowski, P., Joss,P.C., Rappaport, S.: A merger model for SN 1987 A, A\&A 227, L9L12 (1990)

96. Prieto, J.L., Kistler, M.D., Thompson, T.A., Discovery of the Dust-Enshrouded Progenitor of SN 2008S with Spitzer, ApJ 681, 9 (2008)

97. Puls J., Springmann U., Lennon M.: Radiation driven winds of hot luminous stars. XIV. Line statistics and radiative driving, A\&AS 141, 23-64 (2000)

98. Pustilnik, S.A., Tepliakova, A.L., Kniazev, A.Y., Burenkov, A.N.: Discovery of a massive variable star with $\mathrm{Z}=$ Zsolar/36 in the galaxy DDO 68, MNRAS 388, L24-L28 (2008)

99. Ryder, S.D., Sadler, E.M., Subrahmanyan, R., et al.: Modulations in the radio light curve of the Type IIb supernova 2001ig: evidence for a Wolf-Rayet binary progenitor?, MNRAS, 349 , 1093-1100 (2004)

100. Schaller G., Schaerer D., Meynet G., Maeder A.: New grids of stellar models from 0.8 to 120 solar masses at $Z=0.020$ and $Z=0.001$, A\&AS 96, 269-331 (1992)

101. Schulte-Ladbeck, R.E., Clayton, G.C., Hillier, D.J., Harries, T.J., Howarth, I.D.: The axisymmetric stellar wind of AG Carinae, ApJ 429, 846-856 (1994) 
102. Smith, L.J., Nota, A., Pasquali, A., et al.: Ejected Nebulae as Probes of the Evolution of Massive Stars in the Large Magellanic Cloud, ApJ 503, 278 (1998)

103. Smith, N.: Discovery of a Nearby Twin of SN 1987A's Nebula around the Luminous Blue Variable HD 168625: Was Sk -69 202 an LBV?, AJ 133, 1034-1040 (2007)

104. Smith, N., Owocki, S.P.: On the Role of Continuum-driven Eruptions in the Evolution of Very Massive Stars and Population III Stars, ApJ 645, L45-L48 (2006)

105. Smith, N., Davidson, K., Gull, Th.R., Ishibashi, K., Hillier, D.J.: Latitude-dependent effects in the stellar wind of eta Carinae, ApJ 586, 432-450 (2003)

106. Smith N., Vink J.S., de Koter A.: The Missing Luminous Blue Variables and the Bistability Jump, ApJ 615, 475-484 (2004)

107. Smith, N., Li, W., Foley, R.J, et al.: SN 2006gy: Discovery of the Most Luminous Supernova Ever Recorded, Powered by the Death of an Extremely Massive Star like Eta Carinae, ApJ 666, 1116 (2006)

108. Smith, N., Ganeshalingam, M., Chornock, R., et al., ApJ 697, 49 (2009)

109. Soderberg, A.M., Chevalier, R.A., Kulkarni, S.R., Frail, D.A.: The Radio and X-Ray Luminous SN 2003bg and the Circumstellar Density Variations around Radio Supernovae, ApJ 651, 1005-1018 (2006)

110. Stahl, O., Wolf, B.: Circumstellar shells around luminous emission-line stars in the Large Magellanic Cloud, A\&A 158, 371-381 (1986)

111. Stahl, O., Wolf, B., Klare, G., et al.: R 127 - an S DOR type variable intermediate between Of and WN, A\&A 127, 49-62 (1983)

112. Stahl, O., Wolf, B., Klare, G., Juettner, A., \& Cassatella, A.: Observations of the new luminous blue variable R 110 of the Large Magellanic Cloud during an F star-phase, 1990, A\&A 228, 379-386 (1990)

113. Stahl, O., Jankovics, I., Kovács, J., et al.: Long-term spectroscopic monitoring of the Luminous Blue Variable AG Carinae, A\&A, 375, 54-69 (2001)

114. Sterken, C., Wolf, B.: Mass loss of B1 Ia-O supergiants, A\&A 70, 641-651 (1978)

115. Sterken, C., Gosset, E., Juttner, A., et al.: HD160529 - a New Galactic Luminous Blue Variable, A\&A 247, 383-392 (1991)

116. Sterken, C., van Genderen, A.M., Plummer, A., Jones, A.F.: Wra 751, a luminous blue variable developing an S Doradus cycle, A\&A 484, 463-467 (2008)

117. Stothers, R.B., Chin, C.-W.: Dynamical instability as the cause of the massive outbursts in Eta Carinae and other luminous blue variables, ApJ 408, L85-L88 (1993)

118. Trundle C., Lennon D.J.: Understanding B-type supergiants in the low metallicity environment of the SMC II, A\&A 434, 677-689 (2005)

119. Trundle C., Kotak, R., Vink, J.S., Meikle, W.P.S.: SN 2005 gj: Evidence for LBV supernovae progenitors?, A\&A 483, L47-L50 (2008)

120. Umana, G., Buemi, C.S., Trigilio, C., Hora, J.L., Fazio, G.G., Leto, P.: The Dusty Nebula Surrounding HR Car: A Spitzer View, ApJ 694, 697-703 (2009)

121. Valeev, A.F., Sholukhova, O., Fabrika, S.: A new luminous variable in M33, MNRAS 396, 21L-25L (2009)

122. van Boekel, R., Kervella, P., Schöller, M.: Direct measurement of the size and shape of the present-day stellar wind of eta Carinae, A\&A 410, L37-L40 (2003)

123. van Genderen, A.M.: S Doradus variables in the Galaxy and the Magellanic Clouds, A\&A, 366, 508-531 (2001)

124. van Genderen, A.M., Sterken, C.: Light variations of massive stars (alpha Cyg variables). XVIII. The B[e] supergiants S 18 in the SMC and R $66=$ HDE 268835 and R $126=$ HD 37974 in the LMC, A\&A 386, 926-935 (2002)

125. van Marle, A. J., Langer, N., Garcia-Segura, G.: Constraints on gamma-ray burst and supernova progenitors through circumstellar absorption lines. II. Post-LBV Wolf-Rayet stars,A\&A, 469, 948-948 (2007)

126. Vink, J.S.: Constraining GRB progenitor models by probing Wolf-Rayet wind geometries in the Large Magellanic Cloud, A\&A 469, 707-711 (2007)

127. Vink, J.S. de Koter, A.: Predictions of variable mass loss for Luminous Blue Variables, A\&A, 393, 543-553 (2002) 
128. Vink J.S., de Koter A., Lamers H.J.G.L.M.: On the nature of the bi-stability jump in the winds of early-type supergiants, A\&A 350, 181-196 (1999)

129. Vink J.S., de Koter A., Lamers H.J.G.L.M.: New theoretical mass-loss rates of O and B stars, A\&A 362, 295-309 (2000)

130. Vink, J.S., Davies, B., Harries, T.J.: On the presence and absence of disks around O-type stars, A\&A 505, 743 (2009)

131. Walborn, N.R.: Ofpe/WN9 circumstellar shells in the Large Magellanic Cloud, ApJ 256, 452-459 (1982)

132. Weis, K.: On the structure and kinematics of nebulae around LBVs and LBV candidates in the LMC, A\&A 408, 205-229 (2003)

133. White, S.M., Duncan, R.A., Lim, J. et al.: The radio source around Eta Carinae, ApJ 429, 380-384 (1994)

134. Whitelock, P.A., Feast, M.W., Koen, C., Roberts, G., Carter, B.S.: Variability of Eta-Carinae, MNRAS 270, 364-372 (1994)

135. Wolf, B.: Empirical amplitude-luminosity relation of S Doradus variables and extragalactic distances, A\&A 217, 87-91 (1989)

136. Woosley, S.E., Blinnikov, S., Heger, A.: Pulsational pair instability as an explanation for the most luminous supernovae, Nature 450, 390-392 (2007)

137. Yungelson, L. R., van den Heuvel, E.P.J., Vink, J.S., Portegies Zwart, S.F., de Koter, A.: On the evolution and fate of super-massive stars, A\&A 477, 223-237 (2008)

138. Zethson, T., Johansson, S., Davidson, K.; Humphreys, R. M., Ishibashi, K., Ebbets, D.: Strange velocities in the equatorial ejecta of eta Carinae, A\&A 344, 211-220 (1999)

139. Zickgraf, F.-J., Wolf, B., Leitherer, C., Appenzeller, I., Stahl, O.: B(e)-supergiants of the Magellanic Clouds, A\&A 163, 119-134 (1986) 